\title{
Experiences with chest trauma: Where do we stand today
}

\author{
M. Mohta, P. Kumar, A. Mohta*, R. Bhardwaj, A. Tyagi, A. K. Sethi
}

Background and Aims: Chest trauma is a major public health problem in India, but only few studies have been conducted to analyze its magnitude and management. The present study was carried out to determine the epidemiological profile of chest trauma cases and to analyze the management strategies with an aim to identify the scope of improvement in our set-up. Materials and Methods: It is a retrospective study of cases admitted with chest trauma, to a tertiary care hospital. Records of the patients admitted to the hospital with chest trauma over a period of one year were analyzed for the patients' demographic profile; mechanism, nature and severity of injuries; associated injuries; management and outcome of cases. Results: Out of a total of 105 patients, most were males, belonged to age group of 21-40 years and suffered blunt trauma. Motor vehicle accident was the commonest mechanism of injury. The interval from injury to admission ranged from one hour, to more than 24 hours. Eight patients were admitted to the ICU, out of which 5 required ventilatory support. The most frequently used analgesics in the wards were non-steroidal antiinflammatory drugs (NSAID). Ninety one patients improved, while seven patients died in the hospital. Conclusions: Chest trauma due to blunt injury is an important cause of morbidity and mortality in young males. Despite limitations of resources and manpower, attempts are being made to manage patients successfully. However, creation of dedicated trauma teams with well-designed management protocols in hospitals can further improve the outcome.
\end{abstract}

Key words: Chest trauma, fracture ribs, pain relief, pre-hospital care

Trauma is a leading cause of morbidity and mortality all over the world. Chest trauma constitutes about 10$15 \%$ of all the cases and is responsible for $25 \%$ of deaths due to trauma. ${ }^{[1]}$

It is a major problem for India, also due to a very high incidence of vehicular accidents $(6 \%$ of global vehicular accidents), other accidental injuries, crime and violence. ${ }^{[2]}$

\section{From:}

Departments of Anaesthesiology and Critical Care, *Surgery, University College of Medical Sciences and Guru Teg Bahadur Hospital, Delhi, India

\section{Correspondence:}

Dr. Medha Mohta, 28-B, Pocket C, SFS Flats, Mayur Vihar Phase-III, Delhi - 110 096, India. E-mail: medhamohta@hotmail.com
But very few studies have been conducted to assess its magnitude and management in the Indian scenario. Hence the present retrospective study was carried out to determine the epidemiological profile of chest trauma cases and to analyze management strategies with an aim to identify scope of improvement in our set-up.

\section{Materials and Methods}

Records of patients admitted to the hospital with chest trauma over a period of one year, were retrieved from medical records section. The data was analyzed for the patients' demographic profile; mechanism, nature and severity of injuries; associated injuries; management and outcome of cases. The duration of intensive care unit 
(ICU) or hospital stay, type of pain relief provided and any surgical interventions during the course of management, were also recorded. The severity of injuries was assessed by grading different injuries on Abbreviated Injury Scale (AIS) and then calculating Injury Severity Score (ISS). ${ }^{[3]}$

\section{Observations}

A total of 105 patient's with chest trauma were admitted, over a period of one year. Out of these, 95 were males. The maximum number of patients (49.5\%) belonged to the age group of 21 to 40 years. Nine patients were of 65 years or more, in age. Eighty-two patients (78\%) suffered blunt trauma. The most common mechanism of injury was motor vehicle accidents (MVA), followed by falls and assaults [Table 1]. Two patients were hit by animals.

The interval from injury to admission ranged from one hour, to more than 24 hours. Four patients were first taken to private hospitals and were referred to our hospital two or three days after injury.

The patients presented with various types of chest injuries e.g. pneumothorax, haemothorax, haemopneumothorax, fracture ribs, flail chest, and subcutaneous emphysema [Table 2]. Forty nine patients had rib fractures and 14 of these suffered from flail chest. The incidence of lung contusion could not be found out, because of inadequate data availability in the records. Many patients had associated injuries including head injury, spinal cord injury, abdominal visceral injuries and fractures of extremities [Table 2]. ISS ranged from 1 to

\begin{tabular}{lc}
\hline Table 1: Mechanism of injury & \\
\hline Mechanism of trauma & Number of patients \\
Motor vehicle accident & 41 \\
Fall from height & 26 \\
Assault & 21 \\
Gunshot & 4 \\
Industrial accident & 4 \\
Hit by animals & 2 \\
Others & 7 \\
\hline
\end{tabular}

\section{Table 2: Nature of injuries}

\begin{tabular}{lllr}
\hline Type of chest injury & & Associated injuries \\
Fracture ribs & 49 & Haemoperitoneum & 5 \\
Flail chest & 14 & Head injury & 3 \\
Pneumothorax & 49 & Spine injury & 15 \\
Haemothorax & 44 & Faciomaxillary injury & 3 \\
Haemopneumothorax & 36 & Orthopaedic injuries & 13 \\
Subcutaneous emphysema & 44 & & \\
\hline
\end{tabular}

41 for different patients. Eighteen patients had ISS $\leq 8$ and 13 had ISS $\geq 19$. The maximum number i.e. 59 patients, had a worst AIS of 3 for chest injuries; whereas 9, 13, 23 and 1 patient had worst AIS of 1, 2, 4 and 5, respectively. Intercostal drain was inserted in 65 patients, while 17 required other major or minor surgical procedures for management of their injuries.

Eight patients were admitted to the ICU and 5 of them required ventilatory support. The rest of 97 patients were treated in the surgical wards. Out of the 8 patients in ICU, 5 had multiple rib fractures and 3 of these suffered from flail chest. Associated injuries included trauma to abdomen, scalp, spine and other bones. ISS of ICU patients varied from 9 to 32, with four patients having ISS $\geq 19$. Two patients (ISS 17 and 26) expired, of whom one was 65 years old. The rest of the patients were discharged in a satisfactory condition.

Most of the patients received only oral or parenteral, non-steroidal, anti-inflammatory drugs (NSAID) for pain relief. Diclofenac was the most commonly used drug, whereas Ibuprofen, Rofecoxib and Parecoxib were the other NSAIDs used. Only a small minority i.e. six patients received opioids in the form of Morphine and Tramadol. Four patients with flail chest received thoracic epidural analgesia and two were administered intercostal blocks with Bupivacaine. Patients admitted in the ICU received chest physiotherapy along with pain relief and other supportive treatment. But data on such adjunctive therapy was not available in the records of the patients managed in the wards.

The duration of ICU stay ranged from 1 to 30 days, whereas the total hospital stay varied from 1 to 70 days. Ninety one patients improved and were discharged. Four patients left hospital against medical advice, while three absconded. Seven patients died in the hospital. Besides two deaths in ICU, five other patients who were managed in the wards, died. A 95 years old male with a history of fall from height, had spinal fractures, whereas another 80 years old male victim of assault, had multiple fractures along with chest injuries. The other three were young patients who had associated abdominal, spinal and head injuries.

\section{Discussion}

Trauma is a leading cause of morbidity and mortality 
in India. Rising population, accelerated urbanization, industrialization and drastic increase in vehicular transport, have contributed to an annual increase in road traffic accidents by $3 \% .{ }^{[2]}$ An accidental death is reported every 1.9 minutes in India. ${ }^{[4]}$ Although trauma centers have emerged in various parts of the country, trauma care still seems to be at a developmental stage. ${ }^{[2]}$

The present study included only chest trauma patients that were admitted to our hospital, as details of the patients treated on outpatient basis were not available. The data revealed that most of the victims of chest trauma were young males. The incidence of blunt trauma was about $80 \%$. MVA and falls were the most common mechanisms of injury. Similar pattern of injuries has been reported by other workers. ${ }^{[1,5]}$ Two patients in the present series, were injured by animals. One of them was hit by a cow resulting in rib fractures, pneumothorax and subcutaneous emphysema, along with lacerated wound on face. The other patient was injured by a buffalo's horn and developed multiple rib fractures, flail chest and haemopneumothorax. This mechanism of injury is rarely reported in English literature, but is commonly seen in patients from rural areas in India, as animals are frequently used for agriculture and transport. Incidence of penetrating injuries in the present series was low. A higher incidence of about $43 \%$ was reported in an Indian study by Beg et al, ${ }^{[6]}$ which could have been due to a higher prevalence of violence in that region.

Variable mortality rates in chest trauma patients have been reported in literature. ${ }^{[6,7]}$ The same in the present series, was $6.6 \%$. Three out of seven deaths occurred in the elderly age group. This group of patients is believed to have a higher risk of mortality ${ }^{[8,9]}$ because of diminished physiologic reserve and increased prevalence of comorbid conditions. ${ }^{[10]}$

In many cases, there was a delay in reaching the hospital. In Delhi, Centralized Accident Trauma Services (CATS) have 35 well equipped ambulances, which transport trauma victims to the nearest hospital. Police vehicles with personnel trained in basic life support are also actively involved in providing pre-hospital care and transportation of trauma victims. But our hospital is located near the eastern border of Delhi and therefore caters to a large number of patients coming from nearby villages of the neighbouring state, where such facilities are still not adequately available. This may have been responsible for the delay in some cases.

ISS has been used as a criterion for hospital and ICU admission, ${ }^{[5]}$ but in the present series, it was not used as a basis for hospitalization. Hospital admission was based upon subjective assessment of the patient's injuries, by the emergency room physician. Many patients with high ISS scores were managed in the wards. It had not been possible to admit these patients in the ICU, due to shortage of beds and more critical patients getting priority for admission. Lack of protocols for management of such patients and communication among clinicians of different specialties may also have been responsible in some cases. Elderly patients with chest trauma are known to have a higher morbidity and mortality and should be managed aggressively with good pain control and respiratory care, ${ }^{[8]}$ but in the present study, many such patients were treated with routine care in the wards.

Usually, NSAID alone are not considered enough to relieve pain in patients with chest trauma, ${ }^{[11]}$ but these were the most commonly used means of analgesia in the study population. Very few patients received opioid analgesics or regional nerve blocks. This was probably due to management of most of the patients in general ward settings. Pain scores to assess the effectiveness of pain relief measures were not documented in the records, but a large number of patients responded to treatment provided in the surgical wards and were discharged after improvement in their condition. This warrants a prospective study to find out analgesic requirements in Indian population with chest trauma.

Thus in the present audit, although, a large number of patients had a satisfactory outcome; we identified certain deficiencies in the management. There were no protocols for management of chest trauma patients. Record keeping was inadequate and many important details e.g. adjunctive therapy in the wards, pain scores and incidence of lung contusion, could not be found out. Inadequate record keeping could also have been due to lack of definite protocols and patient overload, in emergency services. Some patients who deserved ICU care could not get the same. Mortality rate was calculated to be $6.6 \%$, but outcome was not known for 7 patients who either left hospital against medical advice, or absconded. 
India has a paucity of designated or certified trauma centers and most of the trauma patients are still managed at general hospitals. Our hospital is a tertiary care facility, but the concept of having dedicated trauma teams is still not in practice. Specialists from different specialties are available to manage patients, but the outcome can definitely be improved by a better co-ordination amongst them. This can be achieved by creation of trauma teams, which in turn may require increase in manpower and infrastructure. Thus it is necessary to sensitize the higher administration regarding the need of having designated trauma teams, which should work according to welldesigned protocols for management of trauma patients. These protocols should be tailored according to the needs and the facilities and can be modified with time, as more resources become available.

In conclusion, the present study reveals that chest trauma is most commonly seen in young males, the most frequent mechanism being MVA. Working within constraints of resources and manpower, many patients are being managed successfully, yet there is a scope of improvement. Creation of dedicated trauma teams and ICUs in hospitals can provide better and more efficient care to the trauma victims.

\section{References}

1. Ziegler DW, Agarwal NN. The morbidity and mortality of rib fractures. J Trauma 1994;37:975-9.

2. Joshipura MK, Shah HS, Patel PR, Divatia PA, Desai PM. Trauma care systems in India. Injury 2003;34:686-92.

3. Baker SP, O'Neill B, Haddon W Jr, Long WB. The injury severity score: a method for describing patients with multiple injuries and evaluating emergency care. J Trauma 1974;14:187-96.

4. National Crime Records Bureau. Accidental deaths and suicides in India: New Delhi; 1999.

5. Sirmàh M. Türüt H, Topçu S, Gülhan E, Yazici Ü, Kaya S, et al. A comprehensive analysis of traumatic rib fractures: Morbidity, mortality and management. Eur J Cardiothorac Surg 2003;24:133-8.

6. Beg MH, Siddiqui Z, Ansari MN, Reyazuddin. Penetrating chest trauma: A review of 150 cases. J Indian Med Assoc 1989;87:2035.

7. Dougall AM, Paul ME, Finley RJ, Holliday RL, Coles JC, Duff JH. Chest trauma- current morbidity and mortality. J Trauma 1977;17:547-53

8. Bulger EM, Arneson MA, Mock CN, Jurkovich GJ. Rib fractures in the elderly. J Trauma 2000;48:1040-7.

9. Shorr RM, Rodriguez A, Indeck MC, Crittenden MD, Hartunian S, Cowley RA. Blunt chest trauma in the elderly. J Trauma 1989;29:234-7

10. Bergeron E, Lavoie A, Clas D, Moore L, Ratte S, Tetreault S, et al. Elderly trauma patients with rib fractures are at greater risk of death and pneumonia. J Trauma 2003;54:478-85.

11. Karmakar MK, Ho AM. Acute pain management of patients with multiple fractured ribs. J Trauma 2003;54:615-25. 